\title{
Directional Amplification with a Josephson Circuit
}

\author{
Baleegh Abdo, ${ }^{*}$ Katrina Sliwa, Luigi Frunzio, and Michel Devoret ${ }^{\dagger}$ \\ Department of Applied Physics, Yale University, New Haven, Connecticut 06520, USA
}

(Received 19 February 2013; published 1 July 2013)

\begin{abstract}
Nonreciprocal devices perform crucial functions in many low-noise quantum measurements, usually by exploiting magnetic effects. In the proof-of-principle device presented here, on the other hand, two on-chip coupled Josephson parametric converters (JPCs) achieve directionality by exploiting the nonreciprocal phase response of the JPC in the transmission-gain mode. The nonreciprocity of the device is controlled in situ by varying the amplitude and phase difference of two independent microwave pump tones feeding the system. At the desired working point and for a signal frequency of $8.453 \mathrm{GHz}$, the device achieves a forward power gain of $15 \mathrm{~dB}$ within a dynamical bandwidth of $9 \mathrm{MHz}$, a reverse gain of $-6 \mathrm{~dB}$, and suppression of the reflected signal by $8 \mathrm{~dB}$. We also find that the amplifier adds a noise equivalent to less than 1.5 photons at the signal frequency (referred back to the input). It can process up to 3 photons at the signal frequency per inverse dynamical bandwidth. With a directional amplifier operating along the principles of this device, qubit and readout preamplifier could be integrated on the same chip.

DOI: 10.1103/PhysRevX.3.031001
\end{abstract}

\section{INTRODUCTION}

Reciprocity is one of the basic symmetries in wave physics, in which the source and the detector can be exchanged without changing the transmission coefficient [1]. Breaking this symmetry (nonreciprocity) is particularly useful in amplification. Indeed, the signal source often needs to be protected from noise coming down the amplification chain in reverse. Hence, low-noise measurement chains incorporate nonreciprocal devices such as circulators and isolators. These devices exploit a particular nonreciprocal effect known as Faraday rotation, which relies on ferrites and permanent magnets, in order to distinguish between polarized waves propagating in opposite directions [2].

Circulators and isolators also play a pivotal role in state-of-the-art quantum readout of superconducting qubits that utilize Josephson parametric amplifiers [3-12]. Incorporating these devices as preamplifiers preceding the standard high-electron-mobility transistor amplifier (HEMT) has significantly improved the signal-to-noise ratio. This improvement has allowed one to perform single-shot quantum nondemolition readout of the qubit state, monitor its quantum trajectories in real time, and study backaction effects on the qubit state due to weak and strong measurements [3-5]. However, present Josephson parametric amplifiers operating at the quantum limit, such as the Josephson bifurcation amplifier (JBA)

\footnotetext{
*baleegh.abdo@yale.edu

${ }^{\dagger}$ michel.devoret@yale.edu
}

Published by the American Physical Society under the terms of the Creative Commons Attribution 3.0 License. Further distribution of this work must maintain attribution to the author(s) and the published article's title, journal citation, and DOI.
[6,7], the Josephson parametric amplifier (JPA) [8,9], and the Josephson parametric converter (JPC) [10-12], suffer from a serious limitation. They amplify in reflection even when the device has two ports. Thus, in order to separate between incoming and outgoing signals traveling on the same transmission line, and to protect the qubit from the amplified reflected signal, it is imperative to add a chain of at least two circulators between the qubit and the preamplifier. In the case of the JBA for example, which lacks both spatial and temporal separation between the pump and the signal, these circulators are further needed in order to reject the reflected strong pump. Unfortunately, using such a readout scheme comes at the cost of introducing losses between the cavity-qubit system and the preamplifier, hence adding noise to the processed signal. It also prevents an on-chip integration of the cavity-qubit system with the preamplifier.

For these reasons, there is a growing need for a directional Josephson amplifier that (1) has two ports and works in transmission, (2) is matched to the input, (3) has forward and backward transmissions that are as different as possible (nonreciprocal), and (4) retains the present performances of Josephson parametric amplifiers, i.e., power gain, dynamical bandwidth, added noise, and maximum input power [12].

While the transmission requirement might have a possible engineering solution, for instance, by measuring the idler port in the case of the JPC (which is at a different frequency), or using the two-port version of the JPA [13], matching to the input or achieving nonreciprocity is a pressing challenge.

One example of a superconducting nonreciprocal element is the microstrip SQUID amplifier (MSA) [14-20]. The MSA is biased with a dc current and converts an ac flux signal at the input into an amplified voltage at the output. It suffers from several drawbacks: (1) It dissipates energy on 
chip, (2) it has out-of-band backaction that can affect the system under test, and (3) it is suitable for amplifying lowfrequency signals of hundreds of megahertz; (engineering a matching circuit so that it amplifies with large enough gain and low enough noise above $6 \mathrm{GHz}$ is difficult). It is worthwhile mentioning that a new variation of these amplifiers, known as a superconducting low-inductance undulatory galvanometer (SLUG), has recently been developed [21,22]. The main advantage of these amplifiers is that they provide a more efficient coupling of microwave signals in the gigahertz range to low-impedance SQUIDs.

Another example of a directional element is the traveling-wave parametric amplifier [23]. The new amplifier exploits the nonlinear kinetic inductance of superconducting transmission lines in order to parametrically amplify weak propagating microwave signals. The main disadvantages of this amplification scheme are as follows: (1) It dissipates energy on chip because of the finite resistance of the superconducting films; (2) It does not operate near the quantum limit (the added noise by the amplifier back to the input is about 3.4 photons at $9.4 \mathrm{GHz}$ ); (3) It requires an elaborate microwave engineering in order to inhibit generation of higher harmonics of the pump, limit the amount of dispersion exhibited by the nonlinearity of the device, and suppress gain ripples or reflections that arise from imperfect impedance matching; and (4) It lacks temporal and spatial separation between the signal and the pump tones. A new traveling-wave propagating parametric amplifier based on Josephson junctions has also been reported very recently [24].

In this work, we have implemented and measured a novel Josephson parametric amplifier based on threewave mixing, which satisfies the four requirements of directional amplification outlined above.

\section{CONCEPT AND IMPLEMENTATION}

The basic building block of the new device is the JPC, which is a nondegenerate, dissipationless three-wave mixing amplifier [12]. The JPC consists of two half-wave microstrip resonators, which support two differential fundamental modes, denoted as signal $(S)$ and idler $(I)$. The two resonators, characterized by resonance frequencies $f_{S}^{\text {res }}$ and $f_{I}^{\text {res }}$, and external quality factors $Q_{S}$ and $Q_{I}$, intersect at a Josephson ring modulator (JRM), which is positioned at an rf-current antinode of the resonators. The JRM consists of four nominally identical Josephson junctions, and it is flux biased with $\Phi_{0} / 2$, where $\Phi_{0}=h / 2 e$ is the flux quantum. When operated in the amplification mode, the device is fed by a coherent nonresonant common-mode drive denoted as pump (P) at frequency $f_{P}=f_{1}+f_{2}$, where $f_{1,2}$ are the frequencies of the tones in the signal and idler ports $\left(f_{1}\right.$ and $f_{2}$ lie within the bandwidths of the signal and idler resonators, respectively). The JRM, which functions as a nonlinear medium, mixes the three tones at $f_{1}, f_{2}$, and $f_{P}$ and results in amplification of the tones at $f_{1}$ and $f_{2}$ due to downconversion of pump photons into signal and idler photons. One important advantage of using the JPC as a unit cell in such a device is that the signal, idler, and pump modes, which correspond to orthogonal eigenmodes of the JRM, are well separated, both spatially and temporally [25]. This intrinsic property of the JPC ensures that the quantum system, whose signal we want to amplify, is well protected from pump and idler photons.

By expressing the outgoing wave amplitudes $a^{\text {out }}, b^{\text {out }}$ on the signal and idler ports, given in units of square root of photon number per unit frequency, as a function of the incoming wave amplitudes $a^{\text {in }}, b^{\text {in }}$, one gets, in the frequency domain [12],

$$
\begin{aligned}
& a^{\mathrm{out}}\left[+\omega_{1}\right]=r a^{\mathrm{in}}\left[+\omega_{1}\right]-i \sqrt{r^{2}-1} e^{-i \varphi} b^{\mathrm{in}}\left[-\omega_{2}\right], \\
& b^{\mathrm{out}}\left[-\omega_{2}\right]=r b^{\mathrm{in}}\left[-\omega_{2}\right]+i \sqrt{r^{2}-1} e^{i \varphi} a^{\mathrm{in}}\left[+\omega_{1}\right],
\end{aligned}
$$

where $r=\sqrt{G}$ is the amplitude reflection parameter, $G$ is the power gain of the device at the working point (which depends on the device parameters and on the applied pump amplitude), $\varphi$ is the phase of the pump, and $\omega_{1,2}=2 \pi f_{1,2}$. The first and second terms on the right-hand sides of Eqs. (1) and (2) correspond to reflection-gain and transmission-gain processes, respectively. While the reflection-gain process relates between output and input fields at the same port and frequency, the transmission-gain process relates fields separated by frequency $f_{P}$ across different ports.

Note that the phase acquired in the transmission-gain process is nonreciprocal and depends on the phase of the pump. In a previous theoretical work by our group [26], Kamal et al. suggested that nonreciprocity can be generated by applying a gradient of the pump phase between two active devices. It is important to emphasize, however, that although the nonreciprocal mechanism utilized here is the same, i.e., the gradient of the pump phase, the two nonreciprocal schemes are different. While the scheme presented in Ref. [26] could also be implemented with JPCs, it would, in practice, handle only relatively low-frequency microwave signals $(<0.1 \mathrm{GHz})$ without net gain. The present scheme, on the other hand, provides directionality for microwave signals in the gigahertz range with gain.

The new device shown in Figs. 1(a) and 1(b) consists of two nominally identical JPCs paired together back to back on the same chip. The $\mathrm{JPC}_{1}$ on the left and the $\mathrm{JPC}_{2}$ on the right are coupled via a gap capacitor $C \simeq 20 \mathrm{fF}$ between the two idler resonators, whose resonance frequency is $f_{I}^{\text {res }}=15 \pm 0.1 \mathrm{GHz}$. The other end of the idler resonators is capacitively coupled to a superconducting island that is wire bonded to ground. Thus, the idler resonator is inaccessible through external ports, and its fundamental eigenmode functions as an internal mode of the combined system. The device has two ports [see Fig. 1(c)], i.e., the feedlines of the $S$ resonators of $\mathrm{JPC}_{1}$ and $\mathrm{JPC}_{2}$. 

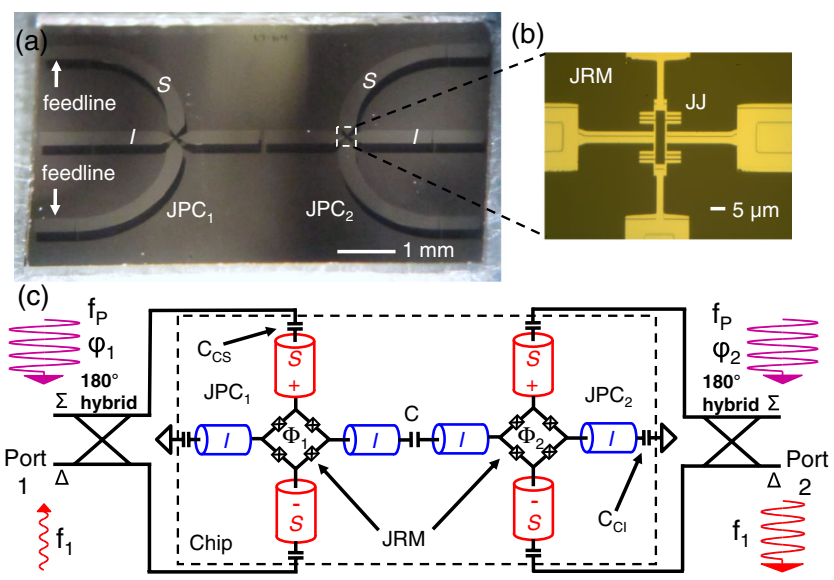

FIG. 1. (a) An optical micrograph of the device, showing two JPCs implemented back to back on the same chip and coupled through their idler resonator using a gap capacitor. The chip size is $8.1 \mathrm{~mm} \times 5.7 \mathrm{~mm}$. The resonators are made of $\mathrm{Nb}$ over a 430- $\mu$ m-thick sapphire substrate. (b) An optical micrograph of one of the JRMs of the device, which consists of four $\mathrm{Al}-\mathrm{AlO}_{x}-\mathrm{Al}$ Josephson junctions. Both JRMs are flux biased near half a flux quantum using two external magnetic coils attached to the copper box housing the device. (c) A circuit representation of the two-port device. The input and output signals are fed and measured through the difference $(\Delta)$ ports of $180^{\circ}$ hybrids connected to $\mathrm{JPC}_{1}$ and $\mathrm{JPC}_{2}$. The JPCs are fed with two independent pump tones at a nonresonant frequency $f_{P}$. The two pumps are injected through the sum $(\Sigma)$ ports of the hybrids and excite a common mode of the JRM. The capacitors $\mathrm{C}_{\mathrm{CS}}$ and $\mathrm{C}_{\mathrm{CI}}$, coupling the $S$ and $I$ resonators to the feedlines and to ground, respectively, are about $38 \mathrm{fF}$ and $30 \mathrm{fF}$. In the case of the $S$ resonators, which are accessible through external ports, this capacitance determines the external quality factor of the device. The coupling capacitor $\mathrm{C}$ between the two $I$ resonators is about $20 \mathrm{fF}$.

At the flux-bias working point, unless indicated otherwise in the text, the $S$ resonators of $\mathrm{JPC}_{1}$ and $\mathrm{JPC}_{2}$ resonate at $f_{S 1}^{\text {res }}=8.47 \mathrm{GHz}$ and $f_{S 2}^{\text {res }}=8.45 \mathrm{GHz}$ with bandwidths of $\kappa_{S 1} / 2 \pi=122 \mathrm{MHz}$ and $\kappa_{S 2} / 2 \pi=116 \mathrm{MHz}$, respectively, which correspond to total quality factors of $Q_{S 1}=69$ and $Q_{S 2}=73$. Similar to the excitation scheme of a single JPC, the $S$ resonators are addressed through the difference $(\Delta)$ port of a $180^{\circ}$ hybrid. The two JPCs are driven by two independent nonresonant pump tones. The pumps share the same frequency $f_{P}$, but they can have different phases $\varphi_{1}$ and $\varphi_{2}$.

The main idea of the device is based on utilizing the nonreciprocal phase response of the JPC in the amplification mode in conjunction with wave interference between multiple paths. These paths are formed, in the device, as a result of the couplings that exist between the idler $I_{1} \leftrightarrow I_{2}$ and signal $S_{1} \leftrightarrow S_{2}$ resonators of the two JPCs. The latter coupling is mediated by a ground plane mode that is enhanced by the idler conductor between the two resonators. Such direct coupling has been experimentally observed by injecting a weak signal through port 1 and measuring the transmission through port 2 (and vice versa) without applying any pump tones. Direct coupling has also been verified using microwave simulations of the device configuration. As a result of the two couplings $I_{1} \leftrightarrow I_{2}$ and $S_{1} \leftrightarrow S_{2}$, feedback loops of the amplified signals are formed between the two JPC stages. Of special importance in this scheme is the transmission-gain process in which signal photons are up-converted to idler and downconverted to signal with gain $\left(S_{1} \leftrightarrow I \leftrightarrow S_{2}\right)$. Because of the nonreciprocal phase response of the JPC, a signal that undergoes sequential transmission-gain processes acquires a nonreciprocal phase shift that depends on the relative phase difference between the pumps. In one direction, it would acquire a phase shift of $\left(\varphi_{1}-\varphi_{2}\right)$, whereas, in the other direction, it would acquire an opposite phase of $-\left(\varphi_{1}-\varphi_{2}\right)$. In contrast, amplified signals that follow a path of direct coupling acquire, regardless of direction, a constant phase shift that depends on the coupling parameters between the resonators and the frequency detuning between the two stages. This detuning can be controlled in situ, to a certain extent, by varying the flux bias threading both JRMs and adjusting it to yield a maximal directional response.

The idea of using a nonreciprocal phase shift in combination with wave interference between two paths has been employed recently in order to demonstrate a photonic Aharonov-Bohm effect at radio frequencies [27]. In Ref. [27], the authors generated a nonreciprocal phase shift on one arm of the interferometer by using rf mixers and controlling the phase difference of the local oscillators feeding them. However, in our work, the amplification aspect, rather than dissipation, is crucial.

\section{DIRECTIONAL AMPLIFICATION}

In Figs. 2(a) and 2(e), we plot power-gain measurements for the four scattering parameters of the device, i.e., $S_{11}$, $S_{21}, S_{12}$, and $S_{22}$, as a function of the relative phase difference between the applied pumps at $f_{P}=23.42 \mathrm{GHz}$. The gain plots drawn in panels (a) and (e), obtained for different flux biases, are measured at $f_{1}=8.447 \mathrm{GHz}$ and $8.468 \mathrm{GHz}$, respectively. In the latter case of panel (e), the resonance frequencies of the two JPCs are $f_{S 1}^{\text {res }}=8.44 \mathrm{GHz}$ and $f_{S 2}^{\text {res }}=8.43 \mathrm{GHz}$. In both measurements, the two pump generators are phase locked to the $10-\mathrm{MHz}$ reference oscillator of a rubidium atomic clock. The phase difference between the pumps is varied in a continuous manner as a function of time, by introducing a frequency offset of a few hertz between the two generators. As can be seen in panel (a) [panel (e)], for a certain relative pump-phase difference, indicated by a vertical dashed line, the device amplifies input signals in transmission $S_{21}$ with a maximum power gain of $15 \mathrm{~dB}(13.6 \mathrm{~dB})$ and attenuates reflections $S_{11}$ by $4 \mathrm{~dB}(12.5 \mathrm{~dB})$. On the other hand, incoming signals in the opposite direction are attenuated in transmission $S_{12}$ by $2 \mathrm{~dB}(19 \mathrm{~dB})$ and amplified in reflection $S_{22}$ by $13 \mathrm{~dB}$ 


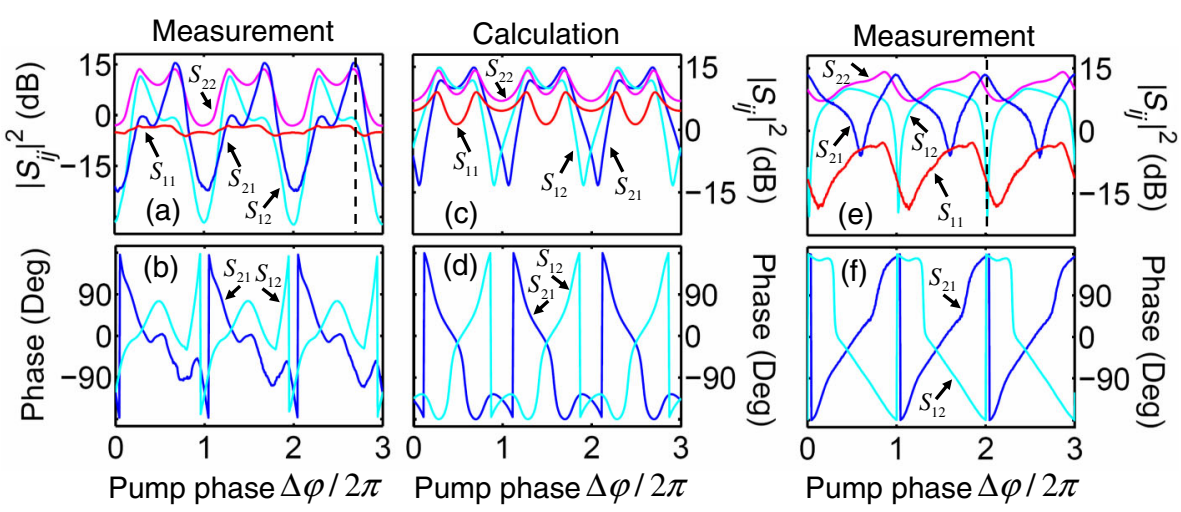

FIG. 2. Panel (a) exhibits measurements of the scattering parameters of the device, namely, $S_{11}, S_{21}, S_{22}, S_{12}$, taken at $f_{1}=8.447 \mathrm{GHz}$, as a function of the phase difference between the pump tones applied at $f_{P}=23.42 \mathrm{GHz}$. The vertical dashed black line indicates a desired working point, at which the device functions as a directional amplifier. Panel (b) plots the nonreciprocal phase response of the device measured in transmission $S_{21}$ and $S_{12}$. Panels (c) and (d) exhibit a theoretical calculation of the scattering parameters of the device using the generic signal flow model shown in Fig. 3. The parameters employed in the calculation are as follows: $\sin \theta_{1}=0.4, \sin \theta_{2}=0.8, \phi_{1}=\pi / 2, \phi_{2}=-\pi / 2, \alpha_{1}=0.6, \alpha_{2}=1, r_{1}=1.5, r_{2}=1.8$. Panels (e) and (f) display an enhanced directional response measured for elevated pump powers. The vertical dashed black line drawn in panel (e) indicates a desired working point. The measurement was taken at $8.468 \mathrm{GHz}$ at a different flux bias than the data shown in panels (a), (b). The asymmetric response of $S_{11}$ and $S_{22}$ with respect to the pump phase is attributed to nonlinear processes that arise at relatively high pump drives.

$(9.5 \mathrm{~dB})$. Note that the amplification in reflection $\left(S_{22}\right)$, despite being generally undesirable, does not influence port 1 (which would potentially be connected to a quantum system). The main differences between the two measurements are as follows: (1) the data in panel (e) display, at the desired working point, a significantly stronger directional response than panel (a), i.e., about $32 \mathrm{~dB}$ difference between $S_{21}$ and $S_{12}$ in panel (e) versus $17 \mathrm{~dB}$ in panel (a); (2) the reflection curves $S_{11}$ and $S_{22}$ in panel (e) exhibit an asymmetrical response with respect to the relative pump phase compared to the curves in panel (a). We attribute the observed asymmetry to nonlinear processes that arise at relatively high pump drives (about $3 \mathrm{~dB}$ higher on both pumps), which are applied in the case of panel (e) compared to panel (a).

In addition to the nonreciprocal amplitude response shown in panels (a) and (e), panels (b) and (f) plot the corresponding nonreciprocal phase of the transmission parameters $S_{21}$ and $S_{12}$.

\section{THEORETICAL MODEL}

In Figs. 2(c) and 2(d), we plot a theoretical calculation based on a generic model of the device schematically drawn in Fig. 3(a). In this model, the two JPCs are effectively coupled together through two-port couplers (beam splitters) $M_{1,2}$, where $M_{1,2}$ connect between the idler and signal ports, respectively. The scattering matrix of the twoport couplers $M_{1,2}$ is of the form

$$
M_{1,2}=\alpha_{1,2} e^{i \phi_{1,2}}\left(\begin{array}{cc}
\cos \theta_{1,2} & i \sin \theta_{1,2} \\
i \sin \theta_{1,2} & \cos \theta_{1,2}
\end{array}\right),
$$

where the angles $\theta_{1,2}$ set the reflection and transmission amplitudes of each coupler, $\phi_{1,2}$ are global phases, and $0<\alpha_{1,2} \leq 1$ are loss factors (for $\alpha_{1,2}=1$, the couplers are lossless). In addition, in order to account for the

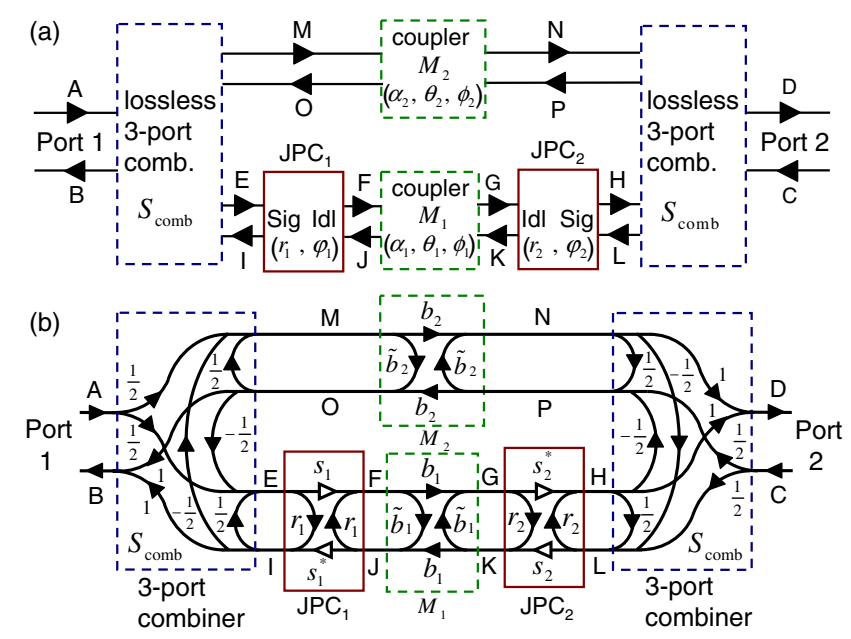

FIG. 3. (a) A generic signal-flow graph modeling the system. The block diagram consists of two JPCs (represented by solid red rectangles) coupled through their idler and signal ports by effective two-port couplers (beam splitters) $M_{1,2}$ (represented by dashed green rectangles). The additional coupling between the signal ports of the device and its external ports 1 and 2 is established by introducing effective three-port combiners or splitters (represented by dashed blue rectangles). (b) A detailed version of the signal graph depicted in panel (a). This graph is used in the derivation of Eqs. (5)-(16), (20), and (21) of the theoretical model. It is also used in the calculation of the device response shown in panels (c) and (d) of Fig. 2. 
coupling between the external ports 1 and 2 of the device and the signal port of each JPC, we assume, for simplicity, a lossless three-port combiner or splitter whose scattering matrix is given by [28]

$$
S_{\text {comb }}=\left(\begin{array}{ccc}
0 & 1 & 1 \\
1 / 2 & 1 / 2 & -1 / 2 \\
1 / 2 & -1 / 2 & 1 / 2
\end{array}\right),
$$

where the scattering parameters of the first row determine the generated output field on the external port 1 or 2 of the device.

To obtain the theoretical curves, amplitude, and phase, plotted in Figs. 2(c) and 2(d), we write the signal flow equations corresponding to the nodes $E$ to $P$, indicated in Fig. 3(b), using Eqs. (1)-(4),

$$
\begin{aligned}
& E-\frac{1}{2} I+\frac{1}{2} O=\frac{1}{2} A, \\
& F-s_{1} E-r_{1} J=0, \\
& G-b_{1} F-\tilde{b}_{1} K=0, \\
& H-s_{2}^{*} G-r_{2} L=0, \\
& I-r_{1} E-s_{1}^{*} J=0, \\
& J-b_{1} K-\tilde{b}_{1} F=0, \\
& K-s_{2} L-r_{2} G=0, \\
& L-\frac{1}{2} H+\frac{1}{2} N=\frac{1}{2} C, \\
& M-\frac{1}{2} O+\frac{1}{2} I=\frac{1}{2} A, \\
& N-b_{2} M-\tilde{b}_{2} P=0, \\
& O-\tilde{b}_{2} M-b_{2} P=0, \\
& P+\frac{1}{2} H-\frac{1}{2} N=\frac{1}{2} C,
\end{aligned}
$$

where

$$
s_{1,2}=-i \sqrt{r_{1,2}^{2}-1} e^{-i \varphi_{1,2}}
$$

and

$$
\begin{gathered}
b_{1,2}=i \alpha_{1,2} \sin \theta_{1,2} e^{i \phi_{1,2},} \\
\tilde{b}_{1,2}=\alpha_{1,2} \cos \theta_{1,2} e^{i \phi_{1,2} .}
\end{gathered}
$$

We also express the output nodes $D$ and $B$ as

$$
\begin{gathered}
D=H+N, \\
B=O+I .
\end{gathered}
$$

By solving the amplitude equations for nodes $E \ldots P$ in response to a unity input signal $A=1$ and $C=0$, we obtain the scattering parameters $S_{21}$ and $S_{11}$ for outputs $D$ and $B$, respectively. Similarly, by solving the same equations for a unity input signal $A=0$ and $C=1$, we obtain the scattering parameters $S_{22}$ and $S_{12}$ for outputs $D$ and $B$, respectively.

Despite the simplicity of the theoretical model that we use to describe the complex dynamics of the system, the calculated curves show a good qualitative agreement with most of the scattering parameter data shown in Figs. 2(a) and 2(b). The main features that are successfully captured by the theoretical model are as follows: (1) the nonreciprocity of the amplitude and phase response of the transmitted signals, $S_{21}$ versus $S_{12}$, (2) the order of magnitude of the scattering parameter amplitudes, (3) the symmetrical response of the scattering parameters with respect to the pump phase difference, and (4) the fact that the reflection parameters $S_{11}$ and $S_{22}$ can be different. On the other hand, the model falls short in yielding exact quantitative agreement with the data shown in panel (a), and in reproducing the asymmetric curve shapes of the scattering parameters, especially $S_{11}$ and $S_{22}$, shown in panel (e) (even for different values of the model parameters). While the former shortcoming can be partly explained by the large parameter space of the theoretical model, which we tried to constrain as much as possible, we attribute the latter shortcoming to nonlinear effects, which come into play at elevated pump powers and are not accounted for in this model.

In addition to explaining the data, it is worthwhile to point out that solving the theoretical model gives some insight into certain requirements for directional amplification. In particular, three main points were uncovered. (1) A finite amount of "loss" between the two JPC stages is required in order to obtain gain and directionality. A possible candidate for loss in our system is power leakage to other modes, for example, leakage to higher-order modes of the system, or to the pump mode, due to finite imbalance between the even and odd modes or a signal-idler photon recombination process [29]. (2) Some imbalance between the reflection parameters of the two stages $r_{1}$ and $r_{2}$ is needed in order to have different $S_{11}$ and $S_{22}$ responses. Such imbalance can be attained in situ by applying different pump amplitudes to the two stages. This finding agrees with the experiment since the observed gains in reflection on the two stages, when pump tones are applied separately, are generally unequal (about $9 \mathrm{~dB}$ on one stage and $2 \mathrm{~dB}$ on the other). (3) Only certain coupling strengths and acquired phases between the two JPCs give rise to directional amplification. This result is in agreement with the experiment as well since we are only able to measure directional 
amplification for certain applied fluxes in the JRMs and pump frequencies. The former control "knob," the magnetic flux, sets the offset between the resonance frequencies of the two stages, while the latter, the pump frequency, determines the range of frequencies $f_{1}$ and $f_{2}$ that get amplified within the bandwidths of the $S$ and $I$ resonators.

\section{DEVICE PERFORMANCES}

Having shown in Fig. 2 that the device satisfies requirements (1), (2), and (3) of directional amplification, namely, the requirements of transmission, input matching, and nonreciprocity, we now set bounds on the device performances, i.e., dynamical bandwidth, added noise, and maximum input power.

In Fig. 4, we plot the device scattering parameters measured as a function of signal frequency at the desired working point, indicated by a vertical dashed line in Fig. 2(a). We find that the 9-MHz dynamical bandwidth of the device, taken at a power gain of $16 \mathrm{~dB}$, is mainly limited by the bandwidth of the signal resonators. Such a dynamical bandwidth is, in general, suitable for qubit-state readout applications, as it exceeds the bandwidths of most readout cavities. Nevertheless, for any practical qubit application, some tunability is required in order to easily match between the center frequency of the amplifier and the readout frequency. One possible way to achieve this tunability is by substituting the present JRM scheme [shown in Fig. 1(b)] by an inductively shunted version similar to the one introduced in Ref. [30]. Incorporating such rings in the next generations of our device is expected to extend the tunable bandwidth to more than $100 \mathrm{MHz}$.

It is also important to note, in the context of this measurement, that although Fig. 4 shows a narrow frequency

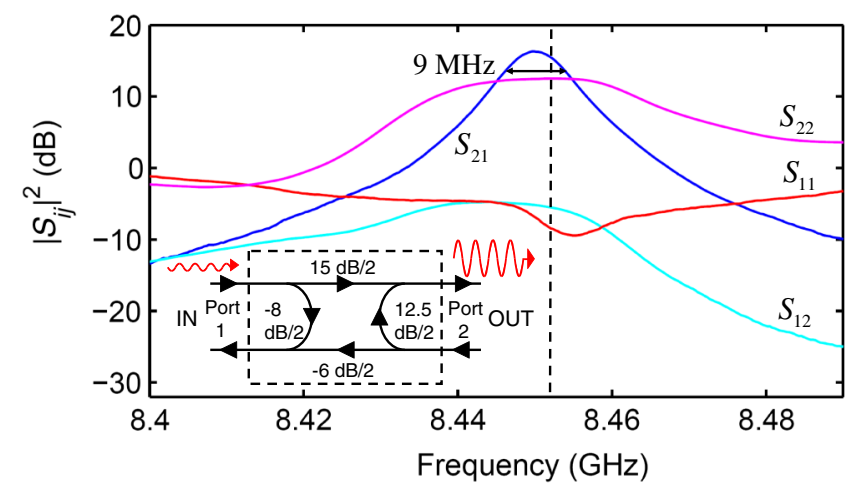

FIG. 4. A measurement of the scattering parameters of the device as a function of signal frequency taken for a fixed pump phase difference that maximizes directionality. The dynamical bandwidth of the device measured at the $3-\mathrm{dB}$ points below the maximum $S_{21}$ gain is $9 \mathrm{MHz}$. In the bottom left part of the figure, we display a schematic sketch of the amplitude gain of the device at $f_{1}=8.453 \mathrm{GHz}$, which corresponds to the dashed vertical line. The pump amplitudes applied in this measurement are the same as in Fig. 2(a).

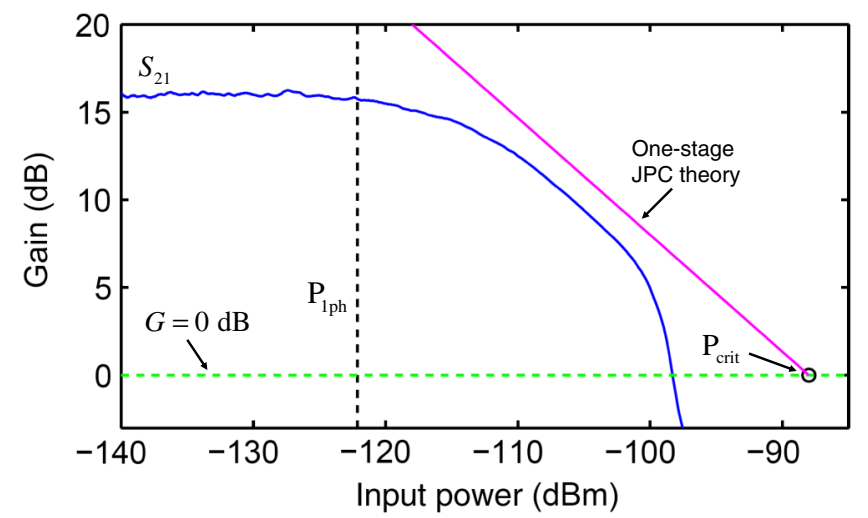

FIG. 5. A measurement of the maximum input power of the device obtained at the desired working point, indicated by a vertical dashed line in Fig. 2(a). The power gain $S_{21}$, drawn in blue, is measured as a function of the input signal power. The vertical dashed black line indicates the input power of one photon at the signal frequency per inverse dynamical bandwidth at $G=16 \mathrm{~dB}$. The solid magenta line is the expected upper limit on the maximum input power of a one-stage JPC, caused by the pump-depletion effect [12].

range around the signal frequency, we have not observed any measurable power leakage at the idler or pump frequencies, when inspecting the power spectrum in a broadband range at the signal ports.

Another important figure of merit for quantum signal amplification is added noise [31]. By measuring the improvement in the signal-to-noise ratio of the system caused by the amplifier and using our knowledge of the noise temperature of the output chain, we are able to set an upper bound on the noise added by the amplifier. Using this measurement method, we find that the device adds up to $1 \pm 0.5$ photons at the signal frequency (referred back to the input), which is similar to the bound we get for single JPC amplifiers [32].

Furthermore, in Fig. 5 we display a maximum-input power measurement taken for $S_{21}$ at the desired working point indicated in Fig. 2(a). The device gain, drawn in blue, is measured as a function of the input signal power. This measurement shows that the device at its maximum gain of $16 \mathrm{~dB}$ can tolerate at least three input photons at the signal frequency per inverse dynamical bandwidth. It also shows that the limiting mechanism on the maximum input power is pump depletion. This can be seen by comparing the gain curve to the magenta line, which depicts the bound on the input power of one-stage JPC due to this effect [12]. A possible improvement of this figure of merit is discussed in Refs. [11,12].

\section{SUMMARY AND FUTURE DIRECTIONS}

We have implemented and measured a proof-ofprinciple, directional Josephson amplifier suitable for qubit readout, which employs a novel nonreciprocal mechanism 
on chip and does not involve magnetic materials. To enhance the device performances, a deeper understanding of the role played by the microwave couplings between the two stages is required. In particular, it should be possible, in principle, to design a coupling scheme between the two stages, which eliminates reflections on both ports of the device at the desired working points, while attaining a high-gain directional amplification. An improved version of this amplifier with respect to microwave control and tunable bandwidth could allow, in the future, on-chip integration with qubit-cavity systems.

Furthermore, one can envision using the same nonreciprocal principles demonstrated in this work, i.e., nonreciprocal phase shift and interference, in order to realize an on-chip four-port circulator, suitable for routing quantum signals. Such a circulator would consist of a gyrator positioned between two hybrids, where the gyrator is a nonreciprocal two-port microwave component that shifts the phase of the transmitted microwave signals by $\pm \pi / 2$, depending on the direction of propagation [2]. However, in this scheme, instead of using a passive gyrator based on magnetic materials, we propose utilizing an active gyrator made of two JPCs coupled through the idler port, with no signal-to-signal coupling, and operated in frequencyconversion mode without photon gain [33]. In this proposed circulator scheme, the active gyrator and hybrids would provide, respectively, the nonreciprocal phase shift and interference paths required for attaining the necessary transmission and isolation between the different ports.

\section{ACKNOWLEDGMENTS}

Discussions with R. J. Schoelkopf, A. Kamal, A. Roy, F. Schackert, and M. Hatridge are gratefully acknowledged. The assistance of M. Power in the fabrication process is highly appreciated. This research was supported by the NSF under Grants No. DMR-1006060 and No. DMR0653377, the NSA through ARO Grant No. W911NF-091-0514, and IARPA under ARO Contract No. W911NF09-1-0369.

\section{APPENDIX: FABRICATION AND MEASUREMENT DETAILS}

The device resonators are made of $\mathrm{Nb}$ over a $430-\mu \mathrm{m}$-thick sapphire substrate. A $2-\mu \mathrm{m}$-thick silver ground plane is e-beam evaporated on the back side of the substrate to enhance thermalization and microwave control. The $\mathrm{Nb}$ layer was patterned using a standard photolithography step and etched using reactive ion etching. The two JRMs of the device were incorporated using a standard e-beam lithography process followed by twoangle shadow evaporation of aluminum (with an oxidation step in between) and liftoff. A large overlap area (partially shown in Fig. 1(b)) is established between the $\mathrm{Nb}$ part of the resonators and the $\mathrm{Al}$ wires of the JRM, which was preceded by plasma cleaning. No measurable losses were observed in our samples because of this interface. The Josephson junction area is $5 \mu \mathrm{m} \times 1 \mu \mathrm{m}$, while the loop area of the JRM is about $50 \mu \mathrm{m}^{2}$. The critical current of the nominally identical Josephson junctions of the JRM is $I_{0}=3 \pm 0.5 \mu \mathrm{A}$.

The measurements are taken in a dilution fridge at a base temperature of $30 \mathrm{mK}$. The experimental setup used is similar to those described in Refs. [11,33], with a few differences. For example, in the present setup, we use two independent pump lines and two magnetic coils. We do not have an idler line (input and output); instead, we use two signal lines (input and output) connected to port 1 and port 2 of the device.

The measurement shown in Fig. 2 is taken using a twoport vector network analyzer (VNA) operated in a zerofrequency span mode centered at $f_{1}$. In order to measure the dependence of the scattering parameters on the relative phase between the two pump tones, we introduce a frequency offset of $10 \mathrm{~Hz}$ between the pump generators and measure 1-s-duration time sweeps on the VNA. The measured time sweeps are externally triggered using an arbitrary wave generator with a periodic signal of $1 \mathrm{~Hz}$, and they yield, as expected, ten periods of the scattering parameter as a function of the pump-phase difference (in Fig. 2, we show only three periods). To ensure a rapid measurement of the different scattering parameters of the device (in order to avoid possible phase drifts between the two microwave generators over time) a room-temperature two-way switch is installed on each port of the VNA, thus allowing fast switching between the input of $\mathrm{JPC}_{1}$ and $\mathrm{JPC}_{2}$, connected to port 1 of the VNA, and between the output of $\mathrm{JPC}_{1}$ and $\mathrm{JPC}_{2}$, connected to port 2. In all measurements, the generators and measuring devices are phase locked to the same $10-\mathrm{MHz}$ reference of a rubidium atomic clock.

[1] R.P. Feynman, QED the Strange Theory of Light and Material (Princeton University Press, Princeton, NJ, 2006).

[2] D. M. Pozar, Microwave Engineering (Wiley, Hoboken, NJ, 2005), 3rd ed.

[3] R. Vijay, D. H. Slichter, and I. Siddiqi, Observation of Quantum Jumps in a Superconducting Artificial Atom, Phys. Rev. Lett. 106, 110502 (2011).

[4] R. Vijay, C. Macklin, D. H. Slichter, S. J. Weber, K. W. Murch, R. Naik, A. N. Korotkov, and I. Siddiqi, Stabilizing Rabi Oscillations in a Superconducting Qubit Using Quantum Feedback, Nature (London) 490, 77 (2012).

[5] M. Hatridge, S. Shankar, M. Mirrahimi, F. Schackert, K. Geerlings, T. Brecht, K. Sliwa, B. Abdo, L. Frunzio, S. Girvin, R. Schoelkopf, and M. Devoret, Quantum BackAction of an Individual Variable-Strength Measurement, Science 339, 178 (2013). 
[6] I. Siddiqi, R. Vijay, F. Pierre, C. M. Wilson, M. Metcalfe, C. Rigetti, L. Frunzio, and M.H. Devoret, RF-Driven Josephson Bifurcation Amplifier for Quantum Measurement, Phys. Rev. Lett. 93, 207002 (2004).

[7] R. Vijay, M.H. Devoret, and I. Siddiqi, The Josephson Bifurcation Amplifier, Rev. Sci. Instrum. 80, 111101 (2009).

[8] M. A. Castellanos-Beltran and K.W. Lehnert, Widely Tunable Parametric Amplifier Based on a Superconducting Quantum Interference Device Array Resonator, Appl. Phys. Lett. 91, 083509 (2007).

[9] T. Yamamoto, K. Inomata, M. Watanabe, K. Matsuba, T. Miyazaki, W. D. Oliver, Y. Nakamura, and J. S. Tsai, FluxDriven Josephson Parametric Amplifier, Appl. Phys. Lett. 93, 042510 (2008).

[10] N. Bergeal, F. Schackert, M. Metcalfe, R. Vijay, V.E. Manucharyan, L. Frunzio, D. E. Prober, R. J. Schoelkopf, S.M. Girvin, and M.H. Devoret, Phase-Preserving Amplification Near the Quantum Limit with a Josephson Ring Modulator, Nature (London) 465, 64 (2010).

[11] B. Abdo, F. Schackert, M. Hatridge, C. Rigetti, and M. H. Devoret, Josephson Amplifier for Qubit Readout, Appl. Phys. Lett. 99, 162506 (2011).

[12] B. Abdo, A. Kamal, and M. H. Devoret, Nondegenerate Three-Wave Mixing with the Josephson Ring Modulator, Phys. Rev. B 87, 014508 (2013).

[13] M. A. Castellanos-Beltran, K. D. Irwin, G. C. Hilton, L. R. Vale, and K. W. Lehnert, Amplification and Squeezing of Quantum Noise with a Tunable Josephson Metamaterial, Nat. Phys. 4, 929 (2008).

[14] M. Mück, J. B. Kycia, and J. Clarke, Superconducting Quantum Interference Device as a Near-QuantumLimited Amplifier at $0.5 \mathrm{GHz}$, Appl. Phys. Lett. 78, 967 (2001).

[15] L. Spietz, K. Irwin, and J. Aumentado, Input Impedance and Gain of a Gigahertz Amplifier Using a dc Superconducting Quantum Interference Device in a Quarter Wave Resonator, Appl. Phys. Lett. 93, 082506 (2008).

[16] M. Mück, D. Hover, S. Sendelbach, and R. McDermott, Microstrip Superconducting Quantum Interference Device Radio-Frequency Amplifier: Effects of Negative Feedback on Input Impedance, Appl. Phys. Lett. 94, 132509 (2009).

[17] M. P. DeFeo, P. Bhupathi, K. Yu, T. W. Heitmann, C. Song, R. McDermott, and B.L.T. Plourde, Microstrip Superconducting Quantum Interference Device Amplifiers with Submicron Josephson Junctions: Enhanced Gain at Gigahertz Frequencies, Appl. Phys. Lett. 97, 092507 (2010).

[18] D. Kinion and J. Clarke, Microstrip Superconducting Quantum Interference Device Radio-Frequency Amplifier: Scattering Parameters and Input Coupling, Appl. Phys. Lett. 92, 172503 (2008).
[19] D. Kinion and J. Clarke, Superconducting Quantum Interference Device as a Near-Quantum-Limited Amplifier for the Axion Dark-Matter Experiment, Appl. Phys. Lett. 98, 202503 (2011).

[20] A. Kamal, J. Clarke, and M.H. Devoret, Gain, Directionality, and Noise in Microwave SQUID Amplifiers: Input-Output Approach, Phys. Rev. B 86, 144510 (2012).

[21] G. J. Ribeill, D. Hover, Y.-F. Chen, S. Zhu, and R. McDermott, Superconducting Low-Inductance Undulatory Galvanometer Microwave Amplifier: Theory, J. Appl. Phys. 110, 103901 (2011).

[22] D. Hover, Y.-F. Chen, G. J. Ribeill, S. Zhu, S. Sendelbach, and R. McDermott, Superconducting Low-Inductance Undulatory Galvanometer Microwave Amplifier, Appl. Phys. Lett. 100, 063503 (2012).

[23] B. H. Eom, P. K. Day, H. G. LeDuc, and J. Zmuidzinas, A Wideband, Low-Noise Superconducting Amplifier with High Dynamic Range, Nat. Phys. 8, 623 (2012).

[24] I. Siddiqi (private communication).

[25] N. Bergeal, R. Vijay, V. E. Manucharyan, I. Siddiqi, R. J. Schoelkopf, S. M. Girvin, and M.H. Devoret, Analog Information Processing at the Quantum Limit with a Josephson Ring Modulator, Nat. Phys. 6, 296 (2010).

[26] A. Kamal, J. Clarke, and M. H. Devoret, Noiseless NonReciprocity in a Parametric Active Device, Nat. Phys. 7, 311 (2011).

[27] K. Fang, Z. Yu, and S. Fan, Experimental Demonstration of a Photonic Aharonov-Bohm Effect at Radio Frequencies, Phys. Rev. B 87, 060301(R) (2013).

[28] Variation on a configuration found in D. M. Pozar, Microwave Engineering (Wiley, Hoboken, NJ, 2005), 3rd ed., pp. 315-323.

[29] F. Schackert, A. Roy, M. Hatridge, A. D. Stone, and M.H. Devoret, Three-Wave Mixing with Three Incoming Waves: Signal-Idler Coherent Cancellation and Gain Enhancement in a Parametric Amplifier, arXiv:1301.1696.

[30] N. Roch, E. Flurin, F. Nguyen, P. Morfin, P. CampagneIbarcq, M. H. Devoret, and B. Huard, Widely Tunable, Nondegenerate Three-Wave Mixing Microwave Device Operating Near the Quantum Limit, Phys. Rev. Lett. 108, 147701 (2012).

[31] C. M. Caves, Quantum Limits on Noise in Linear Amplifiers, Phys. Rev. D 26, 1817 (1982).

[32] See Supplemental Material at http://link.aps.org/ supplemental/10.1103/PhysRevX.3.031001 for a further explanation of the method used to set bounds on the added noise of the amplifier. Also, see Refs. [10], [11], and [31] cited therein.

[33] B. Abdo, K. Sliwa, F. Schackert, N. Bergeal, M. Hatridge, L. Frunzio, A. D. Stone, and M. H. Devoret, Full Coherent Frequency Conversion between Two Propagating Microwave Modes, Phys. Rev. Lett. 110, 173902 (2013). 